\title{
Two-patch model for contact process: migration extends the survival region of species
}

\author{
$\underline{\text { Nariyuki Nakagiri }}^{\text {a }}{ }_{2}$ Kazunori Sato $^{\mathrm{b}}$, Hiroki Yokoi ${ }^{\mathrm{c}}$ and Kei-ichi Tainakab \\ ${ }^{a}$ School of Human Science and Environment, University of Hyogo, Himeji 670-0092, Japan \\ ${ }^{b}$ Department of Systems Engineering, Shizuoka University, Hamamatsu 432-8561, Japan \\ ${ }^{c}$ National Research Institute of Far Seas Fisheries, Japan Fisheries Research and Education Agency, \\ Shimizu, 424-8633, Japan \\ Email: nakagiri@shse.u-hyogo.ac.jp
}

\begin{abstract}
Biospecies usually live in spatially separated patches. Recently, the population dynamics in such patchy environment have been studied extensively. We pay attention to contact process (CP) which is one of simplest ecosystems; both birth and death processes of a single species are carried out on a lattice. In the present paper, we prepare two lattices (patches), and study metapopulation model for CP. Inside respective patches, the birth and death processes of agents (individuals) are carried out. Between patches, agents can migrate. Previously, many authors applied reaction-diffusion equations. Conventional migration method is a diffusion process: migration usually occurs from high-density to low-density patch. However, we apply a refined migration model. A distinct point of the present article is to apply a nonlinear migration; namely, an agent moves into an empty cell. The migration is impossible, if there is no empty cell. We explore the population dynamics depending on mortality rates. If the mortality rate exceeds a critical value $\left(\mu_{\mathrm{c}}\right)$, the species cannot survive. All mean-field models take the same value of $\mu_{\mathrm{c}}$, irrespective of migration rate. In the case of spatial migration model, however, $\mu_{\mathrm{c}}$ takes a higher value with the increase of migration rate. Hence, the survival parameter region of species on lattices is extended by migration.
\end{abstract}

Keywords: Birth and death process, metapopulation, Agent-based model, dynamic phase transition, finite sizes of patches 


\section{INTRODUCTION}

Many biospecies live in spatially separated patches. The population dynamics in such patchy environment is called as metapopulation model in ecology (Hanski, 1999; Hanski and Gilpin, 1997; Levin, 1974). Recently, the metapopulation model has been applied in various fields (Nagatani et al., 2018a; 2018b; Sasmal and Ghosh, 2017), such as epidemiology (Nagatani et al., 2019) and human behaviors (Kabir and Tanimoto, 2019; Wu and Zusai, 2019). In network study, the patches correspond to nodes, and paths connecting patches are thought to be links (Seno, 1998; Nagatani et al 2018a).

In the present article, we deal with two-patch model for contact process (Harris, 1974). An agent- or individualbased model is applied to an ecosystem (Nakagiri et al., 2001; 2005; 2010; Nakagiri and Tainaka, 2004; Chen et al., 2016; Perc, 2011; Szabó and Fath, 2007; Szolnoki and Perc, 2017; Tainaka, 1988; 1989) to take into account the limiting space of patch. Agents live in two patches (lattices), and they randomly migrates between patches. Such a spatial model is very rare in metapopulation researches. Contact process (CP) is extensively studied in various fields, such as mathematics (Harris, 1974; Liggett, 1985) and physics (Marro and Dickman 1999; Katori and Konno, 1991). In epidemiology, CP corresponds to the Susceptible-Infected-Susceptible (SIS) model (Cota et al., 2018; Gross et al., 2006; Peterson, 2010).

We consider CP as an ecosystem; a single species lives on two lattices. Each lattice site is either empty (O) or occupied $(\mathrm{X})$ by an agent. Interactions take place inside respective patches as follows:

$$
\begin{array}{ll}
X+O \stackrel{r}{\longrightarrow} X+X & \text { (rate: } r \text { ), } \\
X \stackrel{\mu}{\longrightarrow} O & \text { (rate: } \mu \text { ), }
\end{array}
$$

Here, reactions (1a) and (1b) denote birth and death processes, respectively. The parameter $r$ and $\mu$ means reproduction rate and death rate, respectively.

\section{PRELIMINARY}

\subsection{One-patch model}

The previous results for $\mathrm{CP}$ are reported. First, we describe the results of one-patch model. We define a patch capacity $(S)$ which means the total number of cells on a lattice. Since $S$ is constant, we put $S=1$. Let $x(t)$ be the density of occupied cell at time $t$. Then the dynamics of system (1) is described by

$$
d x(t) / d t=r x(t)[1-x(t)]-\mu x(t)
$$

The first and second term in the right hand side means birth and death process, respectively (Hofbauer and Sigmund, 1998; Marro and Dickman 1999). Eq. (2) can be rewritten by logistic function:

$$
\frac{d x}{d t}=R x\left(1-\frac{x}{K}\right)
$$

where $R=r-\mu$ and $K=1-\mu / r$. At equilibrium, the positive equilibrium density $\left(x^{*}\right)$ is given by $x^{*}=K$. If we put $r=1$, we have $x^{*}=1-\mu$. When $\mu<1$, the species survives. When $\mu>1$, the species goes extinct. The critical value $\mu_{\mathrm{c}}$ between survival and extinction phases is given by $\mu_{\mathrm{c}}=1$ for wellmixed population. In the case of spatial model, the reaction (1a) occurs between a pair of adjacent cells. For a square lattice, the result of $\mu_{\mathrm{c}} \approx 0.62$ is well known.

\subsection{Conventional two-patch model}

Second, we explain the previous two-patch model for

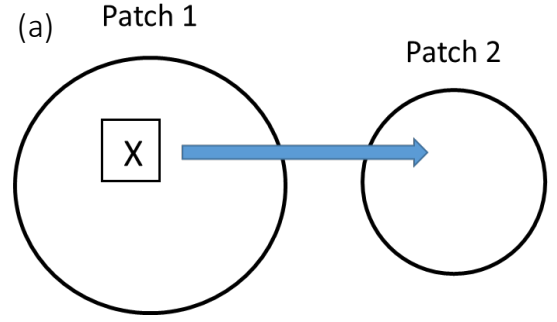

(b) Patch 1

Patch 2

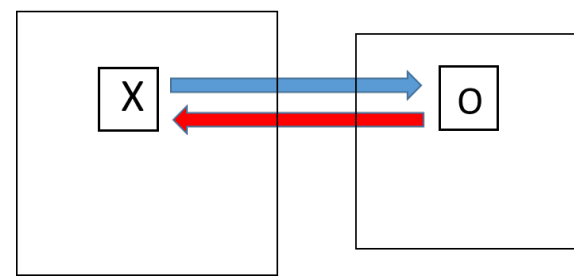

Figure 1. Schematic illustrations of migration methods. (a) Conventional migration, (b) "swapping migration". Subpopulations (patches) are numbered 1 and 2 . We only display the case that an agent $(\mathrm{X})$ migrates from patch 1 to 2 . In (b), the occupied and empty cells are exchanged by migration. If there is no empty cell in patch 2 , any agent in patch 1 cannot migrate.

system (1). An agent freely migrates between patches 1 and 2 [see Fig. 1 (a)]. Let $X_{j}(t)$ be the density of $X$ cell in patch $j$ at time $t$. In addition, $r_{j}$ and $\mu_{j}$ is defined by the reproduction and death rate in patch $j$, respectively. The density is described by 


$$
\frac{d X_{j}}{d t}=R_{j} X_{j}\left(1-\frac{X_{j}}{K_{j}}\right)+m\left[X_{k}-X_{j}\right]
$$

where $R_{j}=r_{j}-\mu_{j}$ and $K_{j}=S_{j}\left(r_{j}-\mu_{j}\right) / r_{j}$. The first and second term in the right hand side means reaction and migration, respectively (Kuang and Takeuchi, 1994; Shigesada and Kawasaki, 1997). The migration term is represented by a linear function of densities (Seno, 1998).

\section{CONTINUOUS TIME STOCHASTIC MODEL}

In Fig. 1(a), a conventional migration is illustrated. In the present paper, however, we apply "swapping migration" (Yokoi et al 2019). As displayed in Fig. 1 (b), the migration is defined by the exchange between occupied (X) and empty (O) cells. Here, we employ periodic boundary conditions. We carried out simulations out as follows:

i) Birth process. We randomly choose a single cell among two lattices. When the chosen cell is $\mathrm{X}$ in patch $i$, then we select one more cell from four adjacent cells in the same patch. If the latter cell is $\mathrm{O}$, it is changed to $\mathrm{X}$ by rate $r_{i}$.

ii) Death process. We randomly choose one cell from two lattices. If the cell is $\mathrm{X}$ in patch $i$, then it becomes $\mathrm{O}$ by rate $\mu_{i}$.

iii) Migration. We randomly choose one cell from two lattices. If the cell is $\mathrm{X}$ in patch $i$, then we choose one more cell in patch $j$. If the latter cell is $\mathrm{O}$, then $\mathrm{X}$ migrate by rate $m$. After the migration, both $\mathrm{X}$ and $\mathrm{O}$ are exchanged.

\section{MEAN-FIELD THEORY}

The dynamics of mean-field theory (well mix population) for $\mathrm{CP}$ of two-patch model with nonlinear migration (Yokoi et al., 2019) is given by

$$
\frac{d X_{j}}{d t}=X_{j}\left[-\mu_{j}+r_{j} e_{j}\right]+m\left[X_{k} e_{j}-X_{j} e_{k}\right]
$$

where $e_{j}$ is the empty density in patch $j$. The first term in the right hand side means death and birth processes in patch $j$. From the simulation method, the birth process occurs in proportion to $e_{j}$ that means the fraction of empty cell within patch $j$. In contrast, $E_{j}$ is the empty density in total both patches $i$ and $j$. Namely, $e_{j}=\left(S_{j}-X_{j}\right) / S_{j}$ and $E_{j}=\left(S_{j}-X_{j}\right) /\left(S_{1}+S_{2}\right)$; thus we obtain $e_{j}=E_{j} / S_{j}$. The last term in Eq. (5) means migration process. From the simulation method, both immigration and emigration terms are proportional not to $E_{j}$ but to $\mathrm{e}_{j}$. It should be emphasized that the migration term in Eq. (5) is represented by nonlinear function of densities. The positive equilibrium density $\left(X_{j}^{*}\right)$ is obtained by setting all the time derivatives in (5) for $j=1, k=2$ and for $j=2, k=1$ to be zero and solving these equations for $X_{1}$ and $X_{2}$ (Yokoi et al., 2019), but it is a little complicated so we omit to give it here.
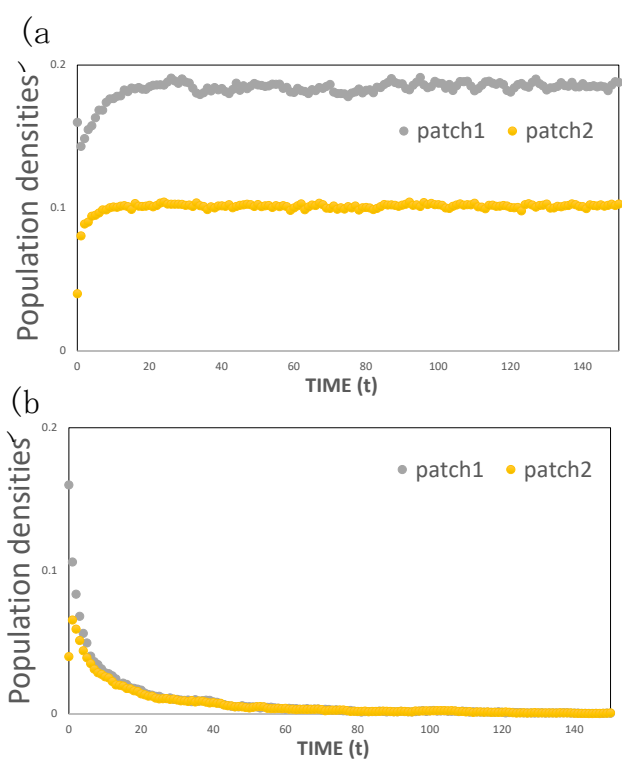

Figure 2. Population dynamics for spatial migration model $\left(r_{1}=r_{2}=1\right)$. The capacity $\left(S_{1}\right)$ of patch 1 is set to be 0.8 : we use $200 \times 200$ and

100x100 lattice for patch 1 and patch 2 , respectively. Initial values are set as $X_{1}=0.16$, $X_{2}=0.04$. (a) Survival phase: $\mu_{1}=\mu_{2}=0.6$, (b) extinction phase: $\mu_{1}=\mu_{2}=0.9$.

Table 1. Parameter lists $\left(r_{j}=1\right)$.

\begin{tabular}{cccc}
\hline & $\mathrm{S}_{1}$ & $\mathrm{~S}_{2}$ & $\mathrm{~m}$ \\
\hline $\begin{array}{c}\text { nonlinear migration of } \\
\text { MFT fig3(a) }\end{array}$ & 0.5 & 0.5 & 1 \\
\hline $\begin{array}{c}\text { nonlinear migration of } \\
\text { MFT fig3(b) }\end{array}$ & 0.8 & 0.2 & 1 \\
\hline $\begin{array}{c}\text { nonlinear migration of } \\
\text { MFT fig3(a) }\end{array}$ & 0.5 & 0.5 & 1 \\
\hline $\begin{array}{c}\text { nonlinear migration of } \\
\text { MFT fig3(b) }\end{array}$ & 0.8 & 0.2 & 1 \\
\hline $\begin{array}{c}\text { spatial model of } \\
\text { fig.4(a) }\end{array}$ & 0.5 & 0.5 & 0 \\
\hline $\begin{array}{c}\text { spatial model of } \\
\text { fig.4(b) }\end{array}$ & 0.8 & 0.2 & 0 \\
\hline $\begin{array}{c}\text { spatial model of } \\
\text { fig.4(c) }\end{array}$ & 0.5 & 0.5 & 1 \\
\hline $\begin{array}{c}\text { spatial model of } \\
\text { fig.4(d) }\end{array}$ & 0.8 & 0.2 & 1 \\
\hline & & & \\
\hline
\end{tabular}


(a) Mean-field theory $S_{1}=0.5$

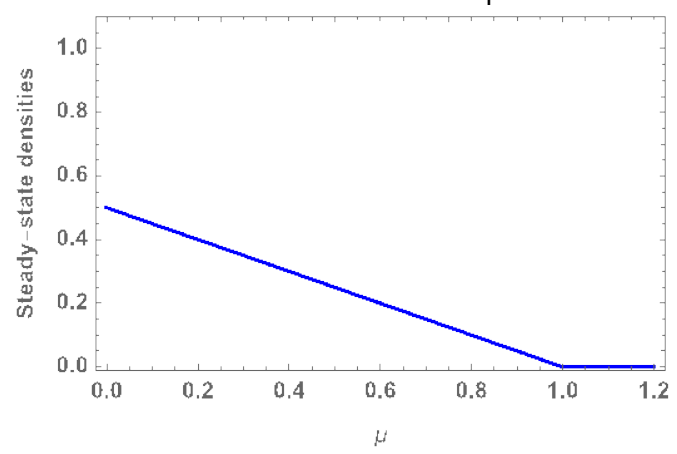

(c) Linear migration $S_{1}=0.5$

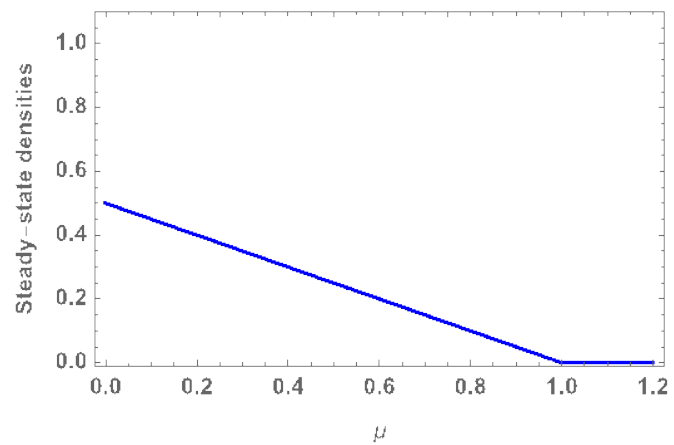

(b) Mean-field theory $S_{1}=0.8$

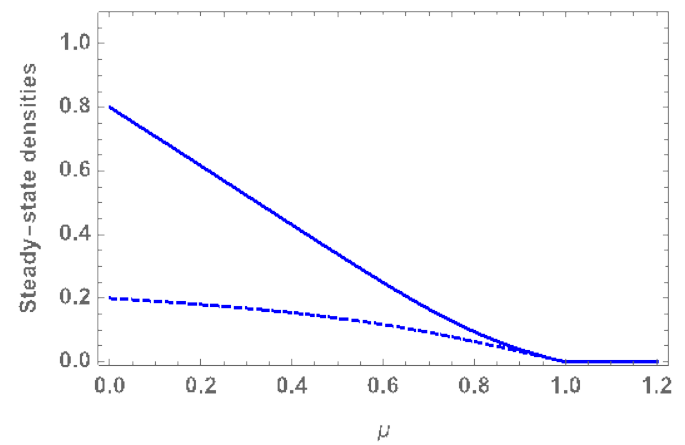

(d) Linear migration $\quad S_{1}=0.8$

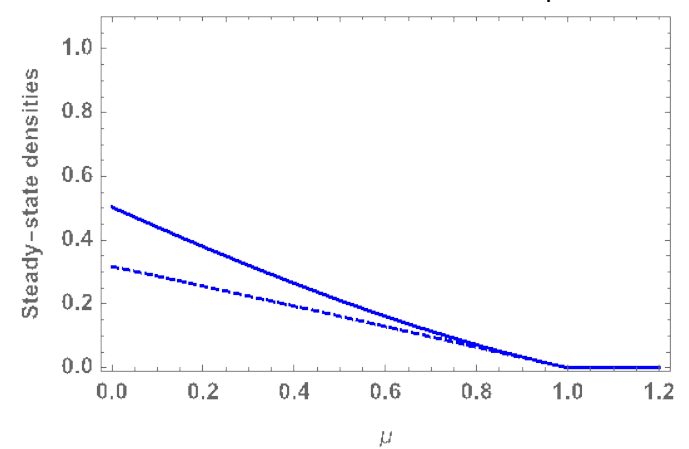

Figure 3. Results for mean-field theory (nonlinear migration). The densities in stationary state are plotted against $\mu$, where we put $\mu_{1}=\mu_{2}=\mu$. The equilibrium densities $X_{j}^{*}$ are numerically obtained at (a) $S_{1}=0.5$ and (b) $S_{1}=0.8$. For the sake of comparison, the results of conventional metapopulation model (linear migration) are displayed in (c) $S_{1}=0.5$ and (d) $S_{1}=0.8$. All non-spatial models exhibit that the phase-transition point is represented by $\mu_{\mathrm{c}}=1$.

\section{RESULTS}

We describe the results for spatial and non-spatial metapopulation models. Non-spatial model is further classified into conventional (linear) and mean-field (nonlinear) migration models. Hereafter, we fix $r_{j}=1$. In Fig. 2, typical population dynamics are plotted for spatial model, where (a) and (b) correspond to survival and extinction phases, respectively. In every case, the system is found to evolve into a stationary state. The final state is either survival or extinction phase.

We explore the densities in stationary state for both spatial metapopulation model and mean-field theory. First, we consider the case that the death rates in both patches take the same value: $\mu_{1}=\mu_{2}=\mu$. The predictions of mean-field theory are described. The equilibrium $X_{j}^{*}$ are numerically obtained. In Fig. 3, the steady-state densities are plotted against $\mu$, where both (a) and (b) represent the cases of mean-field theory (nonlinear migration). For the sake of comparison, the results of conventional metapopulation model (linear migration) are shown in Figs. 3 (c) and (d). The critical value $\mu_{\mathrm{c}}$ between survival and extinction phases is always given by $\mu_{c}=1$ for all non-spatial models. The value $\mu_{\mathrm{c}}=1$ never changes for both migration model $(m>0)$ and onepatch model $(m=0)$.

On the other hand, spatial model shows different behaviors. In Fig. 4, the steady-state densities are plotted against $\mu$ for spatial migration model. Both (a) and (b) are the results for $m=0$, while (c) and (d) are those for $m=1$. In non-migration case $(m=0)$, the critical value takes $\mu_{\mathrm{c}} \approx 0.62$. In contrast, it is found from Fig. 4 (c) and (d) that the survival parameter region is extended by migration; we have $\mu_{c} \approx 0.88$ for $m=1$. Next, we consider the case of $\mu_{1 \neq} \mu_{2}$. The results of mean-field theory are reported. We fix $\mu_{2}=\mu_{\mathrm{c}}=1$, and change the value of $\mu_{1}$. Numerical calculations for $m \geqq 0$ exhibit that the critical value takes $\mu_{1 \mathrm{c}}=1$; namely the species survives for $\mu_{1}<1$ and goes extinct for $\mu_{1} \geqq 1$. Thus, the survival parameter region is always unchanged for mean-field theory. In the case of spatial model, we fix $\mu_{2}=0.88$, and change the value of $\mu_{1}$. Computer simulations for $m=1$ reveal that the critical value takes $\mu_{1 \mathrm{c}} \approx 0.88$. This is the same value for $\mu_{1}=\mu_{2}(m=1)$. 
(a) $m=0, S_{1}=0.5$

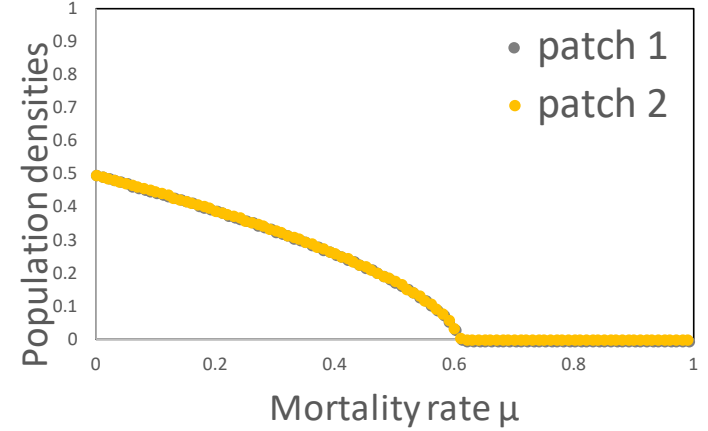

(c) $m=1, S_{1}=0.5$

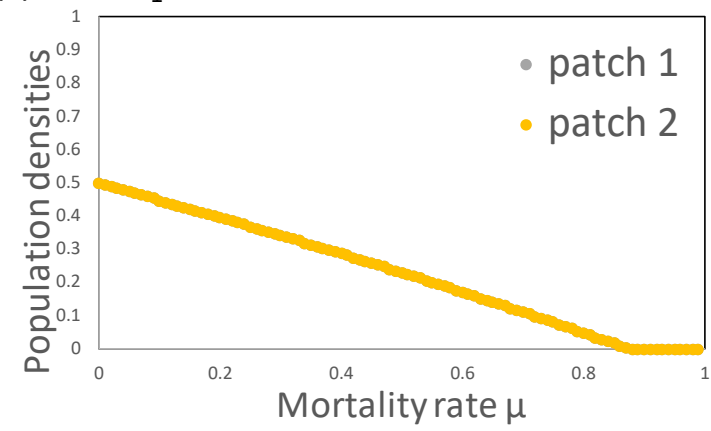

(b) $m=0, S_{1}=0.8$

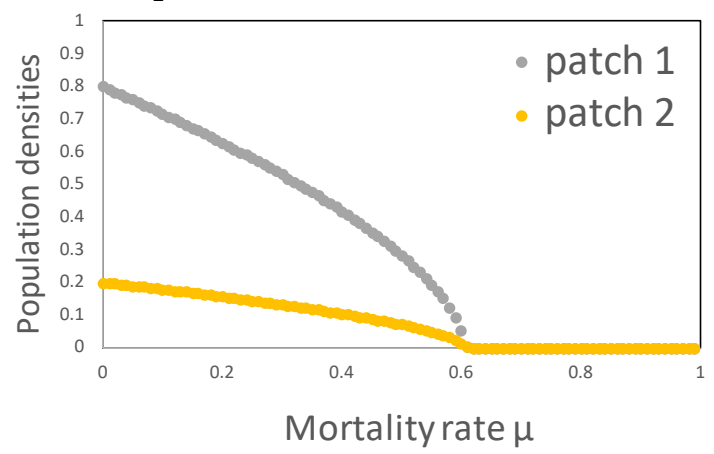

(d) $m=1, S_{1}=0.8$

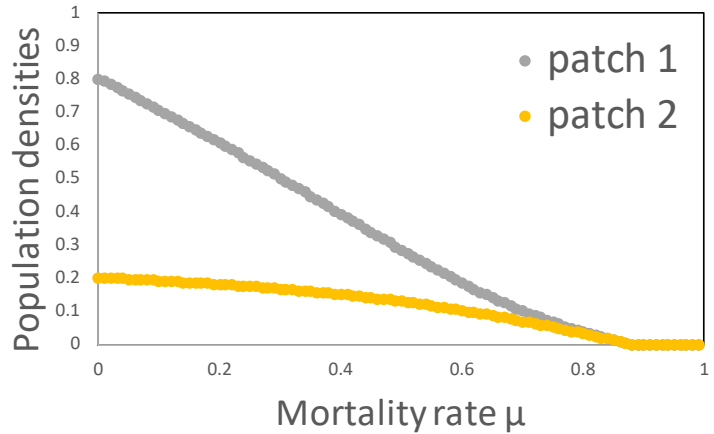

Figure 4. Same as Fig. 3, but for spatial migration model $\left(\mu_{1}=\mu_{2}=\mu\right)$. When migration rate $(m)$ increases, the survival parameter region is extended. (a) $m=0, S_{1}=0.5$, (b) $m=0, S_{1}=0.8$, (c) $m=1, S_{1}=0.5$, (d) $m=1, S_{1}=0.8$. In the case of $S_{1}=0.5$, we use $100 \times 100$ lattice for both patches. On the other hand, when $S_{1}=0.8$, we use $200 \times 200$ and 100x100 lattice for patch 1 and patch 2, respectively.

The spatial pattern formations are explored. In Fig. 5, the spatial distributions in stationary state are displayed near critical points, where (a) $m=0$ and (b) $m=1$. In no-migration case [Fig. 5 (a)], agents aggregate and form clusters. In contrast, agents randomly disperse for $m=1$ [Fig. 5 (b)]. Namely, agents effectively catch the empty (O) by migration. It should be noted that the cell $\mathrm{O}$ is an essential substance (e.g. resource) for birth process (1a).

(a) $m=0$

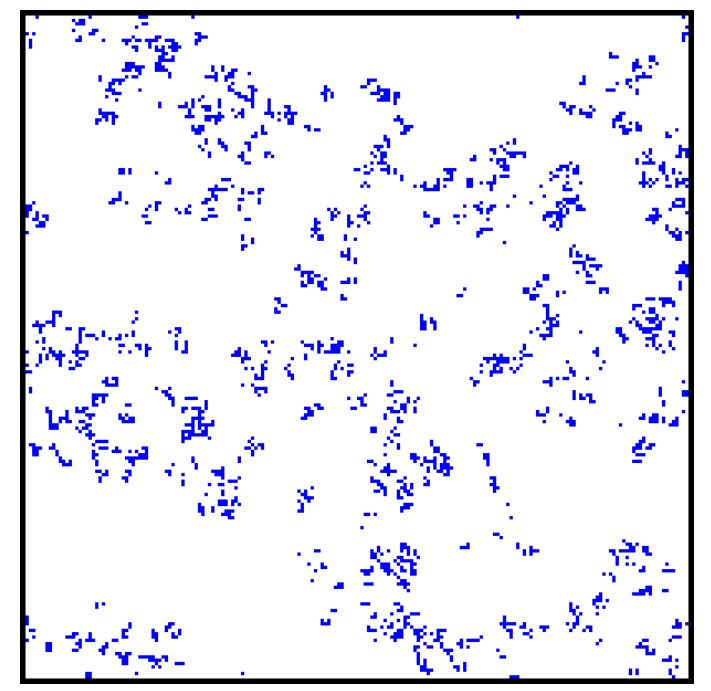

(b) $m=1$

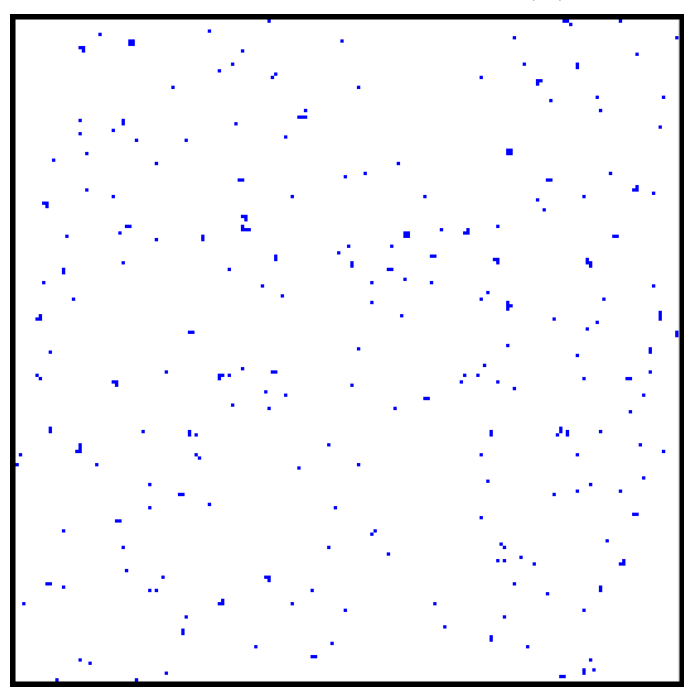

Figure 5. Typical spatial pattern near extinction points. Distributions in patch 1 are displayed $\left(\mu_{1}=\mu_{2}=\mu\right)$. (a) $m=0, \mu=0.6$, (b) $m=1, \mu=0.8$. In non-migration case ( $m=0)$, the species forms clusters (swarms). However, such swarms are collapsed by migration. 


\section{CONCLUSION}

Migration is widely observed in nature (Alerstam, et al., 2003; Shaw, 2016; Yahagi, et al., 2017). In the present article, spatial (agent-based) model is applied to metapopulation dynamics. This lead to "swapping migration" (Yokoi et al 2019). As displayed in Fig. 1 (b), the migration is defined by the exchange between occupied (X) and empty $(\mathrm{O})$ cells. Such a swapping movement is very popular for other transport phenomena. An example is a traffic flow (Gupta et al., 2013; Peng, 2015). A mobile agent (car) cannot move into an occupied cell. When a car moves from occupied to an empty cell, both occupied and empty cells are exchanged. Another example is a random walk of individuals in one-patch ecosystem (Berg, 1993; Sato et al., 2015). Individuals randomly walk, but they cannot go into empty cells. It is found from non-spatial models that the critical value $\left(\mu_{\mathrm{c}}=1\right)$ never changes for both migration $(m>0)$ and non-migration $(m=0)$ models. In contrast, the spatial model shows different behaviors. The critical value takes $\mu \mathrm{c} \approx 0.62$ without migration $(m=0)$, whereas we have $\mu \mathrm{c} \approx 0.88$ with migration $(m=1)$. Hence, the survival parameter region is extended by migration. It is, however, unclear how this work is translated into the understanding of a relevant real world phenomenon.

\section{REFERENCES}

Alerstam, T., Hedenström, A. and Åkesson, S., (2003). Long-distance migration: evolution and determinants. Oikos, 103, 247-260.

Berg, H.C., (1993) Random Walks in Biology. Princeton University Press, New Jersey.

Chen, X., Szolnoki, A. and Perc, M., (2016). Competition of tolerant strategies in the spatial public goods game. New J. Phys., 18, 083021.

Cota, W., Mata, A.S. and Ferreira, S.C., (2018). Robustness and fragility of the susceptible-infected-susceptible epidemic models on complex networks. Phys. Rev. E, 98, 012310.

Gross, T., D'Lima, C.J. and Blasius, B., (2006). Epidemic dynamics on an adaptive network. Phys Rev Lett., 96, 208701.

Gupta, A.K. and Redhu, P., (2013). Analyses of driver's anticipation effect in sensing relative flux in a new lattice model for two-lane traffic system. Physica A, 392, 5622-5632.

Hanski, I., (1999). Metapopulation Ecology. Oxford University Press, New York.

Hanski, I. and Gilpin, M.E., (1997). Metapopulation Biology: Ecology, Genetics, and Evolution. Academic Press, San Diego, CA.

Harris, T.E., (1974). Contact interactions on a lattice. Ann. Probab., 2, 969-988.

Hofbauer, J. and Sigmund, K., (1998). Evolutionary Games and Population Dynamics, Cambridge University Press, Cambridge.

Kabir, K.M.A. and Tanimoto, J., (2019). Evolutionary vaccination game approach in metapopulation migration model with information spreading on different graphs. Chaos, Solitons and Fractals, 120, 41-55.

Katori, M. and Konno, N., (1991). Upper bounds for survival probability of the contact process. J. Stat. Phys., 63, 115-130.

Kuang, Y. and Takeuchi, Y., (1994). Predator-prey dynamics in models of prey dispersal in two-patch environments. Mathematical Biosciences, 120, 77-98.

Levin, S.A., (1974.) Dispersion and population interactions. Am. Nat., 108, 207-228.

Liggett, T. M., (1985). Interacting Particle Systems. New York: Springer.

Marro, J. and Dickman, R., (1999). Nonequilibrium Phase Transition in Lattice Models. Cambridge University Press, Cambridge, New York.

Nagatani, T., Ichinose, G. and Tainaka, K., (2018a). Heterogeneous network promotes species coexistence: metapopulation model for rock-paper-scissors game. Sci. Rep., 8, 7094.

Nagatani, T., Ichinose, G. and Tainaka, K., (2018b). Metapopulation dynamics in the rock-paper-scissors game with mutation: Effects of time-varying migration paths, J. Theor. Biol., 462, 425-431.

Nagatani, T., Ichinose, G. and Tainaka, K., (2019). Epidemic spreading of random walkers in metapopulation model on an alternating graph. Physica A, 520, 350-360. 
Nakagiri et al., Two-patch model for contact process: migration extends the survival region of species

Nakagiri, N. and Tainaka, K., (2004). Indirect effects of habitat destruction in model ecosystems. Ecological Modelling, 174, 103-114.

Nakagiri, N., Tainaka, K. and Tao, T., (2001). Indirect relation between extinction and habitat destruction. Ecological Modelling, 137, 109-118.

Nakagiri, N., Tainaka, K. and Yoshimura, J., (2005). Bond and site percolation and habitat destruction in model ecosystems. J. Phys. Soc. Jpn., 74, 3163-3166.

Nakagiri, N., Sakisaka, Y., Togashi, T., Morita, S. and Tainaka, K., (2010), Effects of habitat destruction in model ecosystems: Parity law depending on species richness. Ecol. Info., 5, 241-247

Nisbet, R.M. and Gurney, W.S.C., (1976). A simple mechanism for population cycles. Nature 263, 319-320.

Olson, R.S. and Knoester, D.B., (2016). Evolution of swarming behavior is shaped by how predators attack. Artif. Life, 318, 299-318.

Peng, G., (2015). Nonlinear analysis of lattice model with the consideration of multiple optimal current differences for two-lane freeway. Modern Physics Letters B, 29, 1550006.

Perc, M., (2011). Does strong heterogeneity promote cooperation by group interactions? New J. Phys., 13, 123027.

Peterson, J., (2010). The contact process on the complete graph with random vertex-dependent infection rates. Stochastic Process its Appl., 121, 609-629.

Sasmal, S.K. and Ghosh, D., (2017). Effect of dispersal in two-patch prey-predator system with positive density dependence growth of preys. Biosystems, 151, 8-20.

Sato, K., Hasegawa, T., Morita, S., Yoshimura, J. and Tainaka, K., (2015). Advantage or disadvantage of migration in a prey-predator system. Far East J. Appl. Math., 93, 109-121.

Seno, H., (1998). Effect of a singular patch on population persistence in a multi-patch system. Ecological Modelling, 43, 271-286.

Shaw, A.K., (2016). Drivers of animal migration and implications in changing environments. Evol. Ecol., 30, 991-1007.

Shigesada, N. and Kawasaki, K., (1997). Biological Invasions: Theory and Practice. Oxford University Press, Oxford, UK.

Szabó, G. and Fath, G., (2007). Evolutionary games on graphs. Physics Reports, 446, 97-216.

Szolnoki, A. and Perc, M., (2017). Second-order free-riding on antisocial punishment restores the effectiveness of prosocial punishment (spatial public goods game). Phys. Rev. X 7, 041027.

Tainaka, K., (1988). Lattice model for the Lotka-Volterra system. J. Phys. Soc. Japan 57, 2588-2590.

Tainaka, K., (1989). Stationary pattern of vortices or strings in biological systems: Lattice version of the LotkaVolterra model. Phys. Rev. Lett. 63, 2688-2691.

Takeuchi, Y., (1996). Global Dynamical Properties of Lotka-Volterra Systems. World Scientific.

$\mathrm{Wu}$, J. and Zusai, D., (2019). A potential game approach to modelling evolution in a connected society. Nature Human Behaviour, 3, 604-610.

Yahagi, T., Kayama, W.H., Kojima, S. and Kano, Y., (2017). Do larvae from deep-sea hydrothermal vents disperse in surface waters? Ecology. 98, 1524-1534.

Yokoi, H., Tainaka, K. and Sato, K., (2019). Metapopulation model for a prey-predator system: Nonlinear migration due to the finite capacities of patches. J. Theor. Biol. 477, 24-35. 